schen nächsten Nachbarn berücksichtigt wird. Dann zeigt sich, wenn man zunächst nur die Funktionen eines Energiebandes des ungestörten Kristalls berücksichtigt, daß außerhalb des regulären Bandes ein "Störband“ auftreten kann mit denselben Eigenschaften, wie sie sich im zweidimensionalen Fall ergaben. Das Ergebnis läßt bei diesem speziellen Modell folgende anschauliche Interpretation zu: Es werden zunächst die Energieniveaus (d.h. Bänder und diskrete Störniveaus) in einer einzelnen $x y$-Ebene berechnet. Setzt man nun viele solcher Ebenen in $z$-Richtung hintereinander, spaltet jedes einzelne Niveau - ähnlich wie bei einer Atomkette - in ein Band auf. Die Bedingung für das Auftreten dieses "Störbandes" und seine energetische Lage hängen jedoch von dem Zahlenwert eines Energieparameters ab, den im wesentlichen bereits Goodwis ${ }^{3}$ für das Auftreten von Oberflächenzuständen an einer eindimensionalen Atomkette herangezogen hat. Die dort angegebene Abschätzung zeigt, daß mit dem Auftreten solcher „Störbänder" außerhalb des regulären Bandes zu rechnen ist. Bei der Berücksichtigung von zwei Bändern, nämlich von Valenz- und Leitungsband des Modellhalbleiters, ergibt sich, daß bei schwacher Wechselwirkung zwischen beiden regulären Bändern die Breite

3 E. T. Goodwin, Proc. Cambr. Phil. Soc. 35, 221 [1939]. Während Goopwis die entsprechenden Matrix-Elemente mit Atom-Eigenfunktionen formulierte, werden sie hier mit Wannier-Funktionen gebildet.

4 Auf die Möglichkeit solcher Störbänder bei Stufenversetzungen im Diamantgitter hat bereits W. Shocklex, Phys. des Störbandes unverändert bleibt, seine energetische Lage aber verschoben wird.

Im vorliegenden wurde zwar nur ein spezielles Modell einer eindimensionalen Gitterstörung untersucht, welches im Realkristall kaum eine Bedeutung haben wird, jedoch darf man wohl die prinzipiellen Ergebnisse auf den allgemeinen Fall übertragen. So wird z. B. bei einer Stufenversetzung mit senkrechter Versetzungslinie die in einer waagerechten Ebene vorliegende Störung in jeder darüberliegenden Ebene wiederholt, so daß auch in diesem Fall bei geeigneter Wahl der Parameter Störbänder ${ }^{4}$ auftreten können, die sich gegenseitig überlappen und den Energiebereich zwischen Valenz- und Leitungsband überdecken können. Die „Störbänder" können dann zur Deutung der strahlungslosen Rekombination an Versetzungen herangezogen werden, da in diesem Fall eine genügende Zahl von erlaubten Energieniveaus vorhanden ist, um den Elektronen des Leitungsbandes durch Wechselwirkung mit den Gitterschwingungen in einer Folge von Einquantenprozessen einen Ưbergang ins Valenzband zu ermöglichen.

Herrn Prof. Dr. Scherzer möchte ich für Anregungen und kritische Diskussionen herzlich danken.

Rev. 91, 228 [1953] hingewiesen. Siehe auch W. C. Dunlap, An Introduction to Semiconductors, New York 1957, S. 189. Vgl. dagegen W. T. READ, Phil. Mag. 45, 775 [1954]; 46, 111 [1955]; Defects in Crystalline Solids, The Physical Society, London 1955, S. 143.

\section{Elektrostatische Aufladung von CdS-Einkristallen unter der Wirkung hoher Felder}

\author{
Von K. W. BöER und U. KüMmeL
}

II. Physikalisches Institut der Humbold-Universität, Berlin, und Laboratorium für die Physik des elektrischen Durchschlags der Deutschen Akademie der Wissenschaften, Berlin (Z. Naturforschg. 12 a, 667-668 [1957] ; eingegangen am 12. Juli 1957)

Werden hohe elektrische Felder an CdS-Einkristalle gelegt so laden sich diese unter Verletzung der Quasineutralitätsbedingung elektrostatisch auf. Rose und SмIтH ${ }^{1,2}$ haben durch Auswerfen des Kristalls aus einer Kontaktzange in die Auffangpfanne eines Elektrometers eine negative Aufladung nachgewiesen und sie als einen experimentellen Hinweis für die Richtigkeit ihrer theoretischen Vorstellungen behandelt. Danach soll eine Injektion von Elektronen aus der Kathode unter Wirkung des elektrischen Feldes und eine Begrenzung des durch den Kristall fließenden Stromes durch ein hierdurch bedingtes Raumladungsgebiet an der Kathode auftreten.

Verf. haben die von Rose und Sмiтн angegebenen Experimente an einer größeren Anzahl von Kristallen fortgeführt und insbesondere die Abhängigkeit der
Aufladung von Dauer und Größe der wirkenden Feldstärke sowie den Einfluß des Elektronenmaterials untersucht. Es wurden dabei die folgenden Resultate erhalten:

1. Die Größe der Aufladung ist entscheidend von der relativen Lage des Kristalls in der Kontaktzange abhängig. Es treten sowohl positive als auch negative Aufladungen auf.

2. Wird der untersuchte Kristall stets möglichst an derselben Stelle in die Kontaktzange gespannt, so streuen die Werte der Aufladungen bedeutend weniger.

3. Es existieren Kristalle, die hinsichtlich einer kleinen Veränderung ihrer Lage in der Kontaktzange sehr empfindlich sind und andere, die auch bei geringfügiger Änderung ihrer Lage noch etwa gleiche Aufladungen zeigen.

4. Eine große Zahl von Kristallen zeigt sowohl positive als auch negative Aufladungen in Abhängigkeit von der Polungsrichtung der angelegten Spannung relativ zum Kristall. Einige Kristalle zeigen nur eine negative, andere Kristalle lediglich positive Aufladung.

5. Die Größe der gemessenen Aufladung nimmt zunächst mit der angelegten Spannung etwa linear zu, durchläuft dann ein Maximum und sinkt dann mit wachsender Spannung ab (vgl. Abb. 1).

6. Die Aufladung ist für Gleichspannungen größer als für Wechselspannungen, welche vor dem Ausklin- 


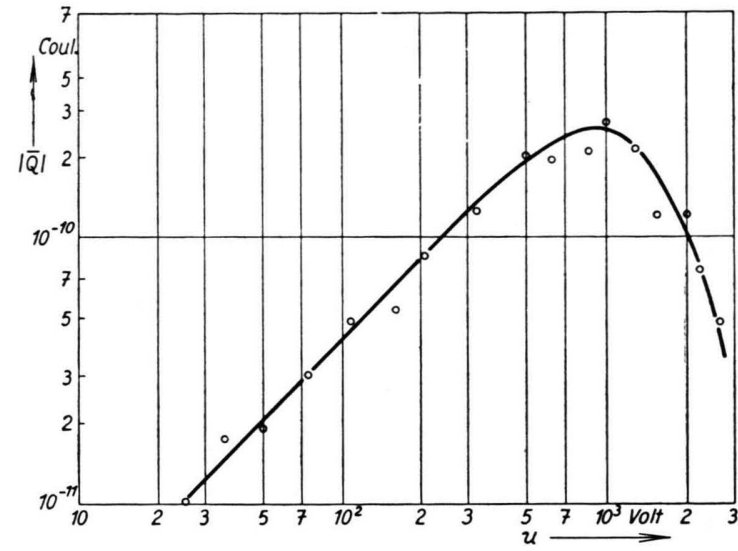

Abb. 1. Aufladung* eines CdS-Einkristalls als Funktion der angelegten Spannung. Dicke des Kristalls 0,2 mm. Kontaktfläche ca. $1 \mathrm{~mm}^{2}$. Die Spannung wurde für eine Dauer von 10 sec an den Kristall gelegt. Der Kristall wurde nicht belichtet. Die Spannung wurde vor dem Auswerfen nicht abgeschaltet.

ken an die Kontaktzange gelegt wurden. Sie nimmt für wachsende Frequenzen rasch ab (vgl. Abb. 2). Dabei erfolgt die Abnahme erst bei höheren Frequenzen, wenn der Kristall in der Kontaktzange schwach belichtet wird.

7. Ein beträchtlicher Einfluß des Kontaktmetalls konnte nicht festgestellt werden.

8. Die Aufladung überlebt ein kurzzeitiges Erden beider Elektroden vor dem Ausklinken.

Diese experimentellen Ergebnisse lassen sich nicht geschlossen nach den Vorstellungen von Rose und Sмiтн deuten. Sie folgen jedoch zwanglos, wenn man annimmt, daß die Leitfähigkeit des CdS-Einkristalls räumlich nicht konstant ist. Dann ergeben sich beim Anlegen hoher Felder dielektrische Nachwirkungen ${ }^{3}$. Die Grenzschichten zwischen besser und schlechter leitenden Bereichen werden je nach der Polung des äußeren Feldes positiv oder negativ aufgeladen.

* Jeder Meßpunkt stellt den Mittelwert des absoluten Betrages von 10 Aufladungsmessungen dar.

3 K. W. Böer u. U. KümmeL, Z. Naturforschg. 9 a, 177 [1954].
Die Aufladungseffekte sind mit relativ hoher Zeitkonstante behaftet, da der Transport der Ladungen über relativ schlecht leitende Kristallbereiche hinweg erfolgt.

$\mathrm{Da}$ in realen Kristallen besser und schlechter leitende Gebiete in regelloser Anordnung verteilt liegen dürften, wird die Größe der Aufladung schwanken je nachdem welche dieser Gebiete im felderfüllten Bereich zwischen den Kontakten liegen.

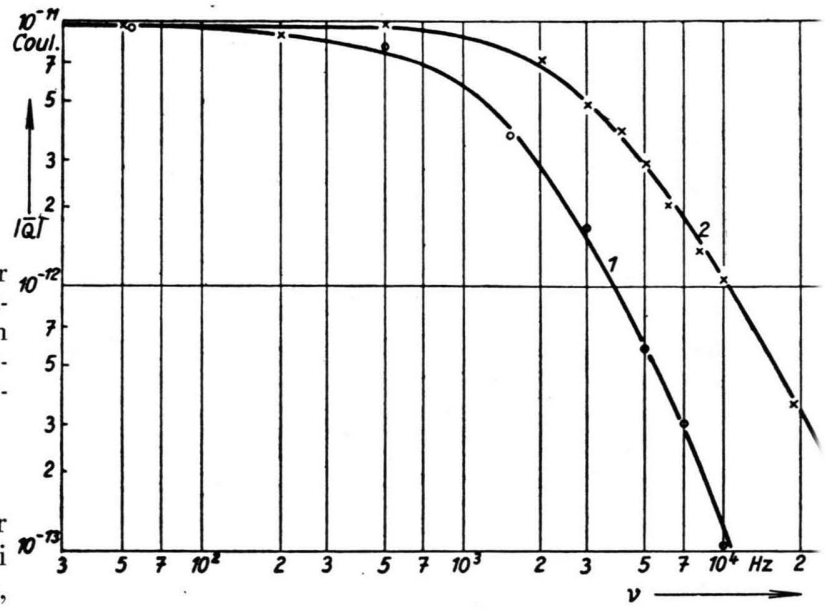

Abb. 2. Aufladung * eines CdS-Einkristalls als Funktion der Frequenz der angelegten Spannung $\left(100 \mathrm{~V}_{\text {eff }}\right)$. Kurve 1 für den unbelichteten Kristall, Kurve 2 bei schwacher Belichtung im Ausläufer $(530 \mathrm{~m} \mu)$.

Mit wachsender wirkender Feldstärke werden die Aufladungen zunächst zunehmen. Im Bereich der Durchschlagsfeldstärke wird jedoch mit einsetzender Feldanregung (vgl. ${ }^{4,5}, 6$ ) eine zunehmende Homogenisierung der Leitfähigekit erfolgen, so daß die Aufladung abnehmen muß.

Im Bereich der Durchschlagsfeldstärke tritt daher der Effekt der dielektrischen Nachwirkung hinter dem der elektrischen Anregung zurück, welcher dort entscheidend den elektrischen Leitungsvorgang bestimmt.

${ }^{4}$ K. W. Böer u. U. Kümmel, Ann. Phys., Lpz. 14, 341 [1954].

5 K. W. Böer u. U. Kümmel, Ann. Phys., Lpz. 16, 181 [1955].

${ }^{6} \mathrm{~K}$. W. W technik, Berlin 1924.

\section{Klärpunkt und Anisotropie der molekularen Polarisierbarkeit kristallin-fluissiger Substanzen}

Von W. Maier und A. SAupe

Physikalisches Institut der Universität Freiburg i. Br. (Z. Naturforschg. 12 a, 668 - 669 [1957] ; eingegangen am 24. Juni 1957)

Seit den chemisch-präparativen Arbeiten VorLÄNDERS ${ }^{1}$ ist bekannt, daß eine mehr oder weniger gestreckte

1 D. Vorländer, Chemische Kristallographie der Flüssigkeiten, Leipzig 1924.
Molekülform (Stabform, Walzenform) Voraussetzung für das Auftreten einer kristallin-flüssigen Phase des nematischen Typs ist. Das einzige molekulare Ordnungsprinzip, dem diese Phasen ihre Anisotropie verdanken, ist das einer Parallelisierung der Moleküllängsachsen ${ }^{2}$. Diese Parallellagerung kann als Nahordnung schon allein durch den Packungseffekt zustande kommen, wie es z. B. die Schüttelversuche von

2 Zusammenfassende Darstellungen über kristallin-flüssige Phasen: W. Kast, Angew. Chem. 67, 592 [1955] ; P. Chatelain, Bull. Soc. franç. Minéral. Cristallogr. 77, 323 [1954]. 